\title{
Phased Technology Application Award
}

National Cancer Institute

\section{Source}

National Cancer Institute. Phased Technology Application Award. NCI Thesaurus. Code C19960.

Similar to Phased Innovation Award, created to support the pilot application of instrumentation, techniques and analytic tools relevant to research on the molecular biology of cancer. (Bypass Budget) 